\title{
Placenta previa: resultados perinatales en la experiencia de una unidad médica de tercer nivel
}

\author{
Placenta praevia: perinatal outcomes in the experience of a third level medical unit
}

Francisco A. Sandoval-García-Travesí, Manuel U. Reyes-Hernández ${ }^{*}$, Dolores Sandoval-Barajas², Sara Morales-Hernández ${ }^{3}$, Valentín Tovar-Galván ${ }^{4}$, Juan C. Hinojosa-Cruz ${ }^{5}$ y

$M^{a}$ Guadalupe Veloz-Martínez

'Servicio de Ginecología y Obstetricia, Unidad Médica de Alta Especialidad, Hospital de Ginecoobstetricia Víctor Manuel Espinosa de los Reyes Sánchez, Centro Médico Nacional La Raza, Instituto Mexicano del Seguro Social (IMSS), Ciudad de México; ${ }^{2}$ Práctica privada, Ensenada, Baja California; ${ }^{3}$ Servicio de Perinatología, Unidad Médica de Alta Especialidad, IMSS, Ciudad de México; ${ }^{4}$ Servicio de Tococirugía, Unidad Médica de Alta Especialidad, Hospital General de Ginecoobstetricia, Centro Médico Nacional, IMSS, Ciudad de México; ${ }^{5}$ Dirección de Educación e Investigación en Salud, Unidad Médica de Alta Especialidad, Hospital General de Ginecoobstetricia, Centro Médico Nacional, IMSS, Ciudad de México; ${ }^{6}$ División de Investigación en Salud, Unidad Médica de Alta Especialidad, Hospital General de Ginecoobstetricia, Centro Médico Nacional, IMSS, Ciudad de México. México

\section{Resumen}

Objetivo: Describir los resultados perinatales de pacientes que cursaron con embarazo complicado con inserción anómala de la placenta. Método: Se revisaron los expedientes clínicos con diagnóstico de inserción anómala de la placenta después de las 34 semanas de gestación de enero de 2012 a enero de 2015. Resultados: Se incluyeron 200 casos. El diagnóstico se realizó mediante ultrasonido endovaginal. El 52.5\% de los casos correspondieron a placenta central total, el $19.5 \%$ a placenta con inserción baja, el 18.5\% a placenta marginal y el $9.5 \%$ a placenta con inserción parcial. El 24\% de los embarazos se resolvieron entre las semanas 34 y 35.6, y el 76\% en la semana 36 o más. Todas las pacientes fueron sometidas a cesárea (el 77\% fueron cirugías programadas y el 23\% cirugías de urgencia). Ameritaron histerectomía obstétrica 77 pacientes. En 58 pacientes se confirmó acretismo por patología. No se registraron muertes maternas. Las complicaciones más frecuentes de los neonatos fueron síndrome de distrés respiratorio (18.5\%) y taquipnea transitoria del recién nacido (5\%). Hubo una muerte neonatal. Conclusiones: El tratamiento quirúrgico de la inserción anómala de la placenta realizado en hospitales de tercer nivel puede prevenir resultados perinatales adversos. Es posible el manejo expectante en pacientes seleccionadas.

PALABRAS CLAVE: Hemorragia obstétrica. Histerectomía obstétrica. Placenta acreta.

\begin{abstract}
Objective: To describe the perinatal results of patients who underwent complicated pregnancy with anomalous insertion of the placenta. Method: We reviewed the clinical records with diagnosis of anomalous placental insertion after 34 weeks of gestation from January 2012 to January 2015. Results: 200 cases were included. Diagnosis was made by endovaginal ultrasound, and $52.5 \%$ of the cases corresponded to total central placenta, $19.5 \%$ to placenta with low insertion, $18.5 \%$ to marginal placenta, and $9.5 \%$ to partial placental insertion. $24 \%$ of the pregnancies were resolved between weeks 34 and 35.6 , and $76 \%$ in week 36 or more. All patients underwent cesarean: $77 \%$ were scheduled surgeries and $23 \%$ emergency surgeries. 77 patients required obstetric hysterectomy. In 58 patients, confirmed accretism by pathology. There were no maternal deaths. The most frequent complications in neonates were respiratory distress (18.5\%) and newborn transitory tachypnea (5\%). The-
\end{abstract}

\author{
Correspondencia: \\ *Manuel U. Reyes-Hernández \\ Vallejo, 266 y 270 \\ La Raza, Azcapotzalco \\ C.P. 2990, Ciudad de México, México \\ E-mail: ulises.reyes.hdz@gmail.com
}

Fecha de recepción: 31-10-2018

Fecha de aceptación: 02-05-2019

DOI: 10.24875/CIRU.19000861
Cir Cir. 2019;87:667-673 www.cirugiaycirujanos.com 
re was one neonatal death. Conclusions: Surgical treatment of anomalous placement of the placenta in third-level hospitals can prevent adverse perinatal outcomes. Expectant management is possible in selected patients.

KEY WORDS: Obstetric hemorrhage. Obstetric hysterectomy. Placenta accrete.

\section{Introducción}

La placenta previa (PP) es una complicación obstétrica que se presenta clásicamente como un sangrado vaginal asintomático en el tercer trimestre. Se debe a una implantación placentaria en el segmento uterino bajo, ya sea cerca o con algún grado de cobertura del orificio cervical interno, y el método de referencia para su diagnóstico es el ultrasonido endovaginal'. La incidencia anual de PP en los EE.UU. es de 2.8 a 4.8 por cada 1000 partos en embarazos únicos y de 3.9 por cada 1000 partos en embarazos múltiples, mientras que la prevalencia global es de 5.2 por cada 1000 nacimientos ${ }^{1-7}$.

La inserción anómala de la placenta constituye un grupo de condiciones clínicas que, en México, en los últimos años, han tomado gran relevancia puesto que prácticamente igualan e incluso han llegado superar a los trastornos hipertensivos del embarazo como causa principal de mortalidad en nuestro país ${ }^{8}$. El acretismo placentario es una inserción anómala de las vellosidades de la placenta en el miometrio. El riesgo de placenta acreta en las mujeres con PP según su historia obstétrica es: $1-5 \%$ sin cirugía uterina previa, $11-25 \%$ con una cesárea previa, $35-47 \%$ con dos cesáreas previas, $40 \%$ con tres cesáreas previas y $50-67 \%$ con cuatro o más cesáreas ${ }^{9-13}$. En México, alrededor del $32 \%$ de las pacientes con PP podrán presentar placenta acreta $^{14}$.

La PP como causa de hemorragia obstétrica es una de las complicaciones más comunes y uno de los problemas clínicos más controvertidos a los que se enfrenta el obstetra, por las diversas opiniones internacionales en cuanto a la manera del abordaje diagnóstico y terapéutico.

Se aceptó en un consenso general que hay una posibilidad razonable de parto vaginal con PP siempre y cuando no haya hemorragia, no se sospeche acretismo por ultrasonido, el borde placentario esté a más de $20 \mathrm{~mm}$ del orificio cervical interno y no existan contraindicaciones para el parto vaginal; de lo contrario, se resolverá mediante cesárea por indicación obstétrica $^{15}$.

La PP en el Centro Médico Nacional La Raza es una de las principales causas de interrupción del embarazo por vía abdominal, la cual puede ser de manera urgente o electiva. Sin embargo, la evidencia científica muestra resultados perinatales adversos en productos pretérmino. El objetivo de esta investigación es describir los resultados perinatales de pacientes que cursaron un embarazo complicado con inserción anómala de la placenta atendidas en nuestra unidad médica.

\section{Método}

Estudio descriptivo retroprolectivo realizado en el Hospital de Ginecoobstetricia número 3 del Centro Médico Nacional La Raza. Se revisaron los expedientes de pacientes con diagnóstico de inserción anómala de la placenta, con embarazo resuelto después de las 34 semanas confirmadas por ultrasonido, en el periodo de enero de 2012 a enero de 2015. Se descartaron expedientes con información insuficiente 0 de pacientes con resolución del embarazo fuera de la institución. Se registraron las variables edad materna, gestaciones, partos, cesáreas, abortos y edad gestacional en el momento de la interrupción, presencia y tipo de complicaciones, indicaciones de cesárea y resultados perinatales. Se utilizó estadística descriptiva para las variables numéricas: medias, medianas y desviación estándar; para las variables cualitativas se utilizaron porcentajes y proporciones. Se utilizaron las pruebas ji al cuadrado y $t$ de Student para comparar variables nominales y numéricas, respectivamente.

\section{Resultados}

Se identificaron e incluyeron 200 pacientes con diagnóstico de inserción anómala de la placenta diagnosticada por ultrasonido. La media de edad de las pacientes en el momento de la intervención fue de 32 \pm 5.36 años (mínimo 17 y máximo 44 años). Las pacientes tenían el antecedente de entre una y ocho gestas, con una media de tres gestas. Eran primigestas $25(12.5 \%)$, secundigestas $60(30 \%)$ y tenían de tres a ocho gestaciones 115 (57.5\%). En el momento de la intervención contaban con el antecedente de entre una y cinco cesáreas, con una mediana de dos. La media de la edad gestacional al interrumpir el 
embarazo según la última menstruación fue de 36.4 semanas (mínimo 34 y máximo 40.2 semanas). La edad gestacional fue verificada al nacimiento por $\mathrm{Ca}$ purro. Se resolvieron 48 embarazos entre las semanas 34 y 35.6, y 152 se resolvieron con 36 o más semanas. A todas las pacientes incluidas en el estudio se les realizó ultrasonido endovaginal y se encontraron datos de acretismo placentario en el 30\% (60 pacientes).

\section{Tipo de inserción placentaria y cirugías realizadas}

A todas las pacientes se les practicó cesárea. El $77 \%(n=154)$ fueron cesáreas programadas y el $23 \%$ $(n=46)$ fueron de manera urgente. En el transoperatorio se observaron datos de acretismo por clínica en $58(29 \%)$ pacientes y 54 de ellas ya contaban con el antecedente de acretismo por ultrasonido.

El tipo de inserción anómala de la placenta que se encontró con mayor frecuencia fue la placenta central total; el tipo de inserción placentaria se resume en la tabla 1.

Veintitrés pacientes (11.5\%) refirieron ser primigestas, ocho con placenta central total y siete con inserción marginal.

De las 200 pacientes incluidas en el estudio, el $38.5 \%$ (77) ameritaron histerectomía obstétrica y los resultados histopatológicos fueron: placenta acreta en el $27.3 \%$ (21), placenta increta en el $27.3 \%$ (21), placenta percreta en el $21 \%$ (16) y sin datos de acretismo en el $24.6 \%$ (19).

Las cirugías programadas fueron más frecuentes en las pacientes con embarazo de 36 o más semanas. Los motivos de la cirugía de urgencia se aprecian en la tabla 2. Los tipos de intervenciones quirúrgicas realizadas se muestran en la tabla 3.

Se realizó ligadura de las arterias hipogástricas a 57 pacientes, sin registrar complicaciones en los procedimientos.

\section{Resultados maternos}

El $15.5 \%$ (31) de las pacientes requirieron ingreso en la unidad de cuidados intensivos del adulto tras la cirugía. De las 200 pacientes, el $2.5 \%$ (5) fueron reintervenidas por sangrado. En el $6.5 \%$ (13) de las pacientes hubo lesión de órgano adyacente, y la más frecuente fue en la vejiga. El sangrado quirúrgico reportado varió de 300 a $10,000 \mathrm{ml}$, con una media de $1506 \mathrm{ml}$. No hubo diferencia estadística al comparar
Tabla 1. Tipo de inserción placentaria por ultrasonido

\begin{tabular}{lcc}
\hline Tipo de inserción anómala de placenta & Total & Porcentaje \\
\hline Inserción baja de placenta & 39 & 19.5 \\
Placenta previa marginal & 37 & 18.5 \\
Placenta previa parcial & 19 & 9.5 \\
Placenta previa central total & 105 & 52.5 \\
Total & 200 & 100 \\
\hline
\end{tabular}

Tabla 2. Indicación de cirugía de urgencia según las semanas de gestación

\begin{tabular}{lccc}
\hline $\begin{array}{l}\text { Indicación de cirugía de } \\
\text { urgencia }\end{array}$ & \multicolumn{2}{c}{$\begin{array}{c}\text { Semanas de gestación } \\
\text { según fecha de última } \\
\text { menstruación }\end{array}$} & Total \\
\cline { 2 - 3 } & Menos de 36 & 36 o más & \\
\hline $\begin{array}{l}\text { Alteraciones en la frecuencia } \\
\text { cardiaca fetal }\end{array}$ & 1 & 2 & 3 \\
Alteraciones del líquido & 0 & 3 & 3 \\
amniótico & 3 & 1 & 4 \\
Desprendimiento de placenta & 3 & 1 & 4 \\
Otras & 5 & 14 & 19 \\
Trabajo de parto & 9 & 4 & 13 \\
Sangrado vaginal persistente & 21 & 25 & 46 \\
\hline Total & & & 3 \\
\hline
\end{tabular}

Tabla 3. Tipos de cirugías realizadas

\begin{tabular}{lcc}
\hline Cirugía & Total & Porcentaje \\
\hline Cesárea & 99 & 49.5 \\
Cesárea+LBAU & 9 & 4.5 \\
Cesárea+LBAH & 5 & 2.5 \\
Cesárea+B-Lynch & 10 & 5 \\
Cesárea+HTA & 25 & 12.5 \\
Cesárea+LBAU+HTA+LBAH & 4 & 2 \\
Cesárea+LBAU+LBAH & 1 & 0.5 \\
Cesárea+HTA+LBAH & 41 & 20.5 \\
Cesárea+HTA+LBAH+empaquetamiento & 6 & 3 \\
Total & 200 & 100 \\
\hline
\end{tabular}

HTA: histerectomía total abdominal; LBAH: ligadura bilateral de arterias hipogástricas; LBAU: ligadura bilateral de arterias uterinas.

la pérdida sanguínea según la edad gestacional o a la realización de cirugía programada o de urgencia. La cantidad de pérdida hemática de acuerdo con la edad gestacional o la programación de la cirugía se muestra en la tabla 4. Ameritaron hemotransfusión el 
Tabla 4. Pérdida sanguínea por semanas de gestación y tipo de cirugía

\begin{tabular}{|c|c|c|c|c|c|c|}
\hline \multicolumn{4}{|c|}{ Semanas de gestación } & \multicolumn{3}{|c|}{ Tipo de cirugía } \\
\hline Pérdida sanguínea & Menos de 36 & 36 o más & Total & Urgencia & Programada & Total \\
\hline$<750 \mathrm{ml}$ & 15 & 70 & 85 & 23 & 62 & 85 \\
\hline$>750 \mathrm{ml}$ & 20 & 43 & 63 & 15 & 48 & 63 \\
\hline$>1500 \mathrm{ml}$ & 3 & 8 & 11 & 2 & 9 & 11 \\
\hline $2000 \mathrm{ml}$ & 10 & 31 & 41 & 6 & 35 & 41 \\
\hline Total & 48 & 152 & 200 & 46 & 154 & 200 \\
\hline
\end{tabular}

$32 \%$ (64) de las pacientes. Requirieron de 1 a 3 paquetes globulares el $37.6 \%$ (26) de las pacientes, el $50 \%$ (32) requirió de 4 a 6 paquetes globulares, el $6.3 \%$ (4) de 7 a 9 paquetes globulares, y el $3.1 \%$ (2) de 10 a 12 paquetes globulares.

La media de estancia hospitalaria de las pacientes fue de 16.07 días (mínimo 2, máximo 52).

No ocurrió ninguna muerte materna durante el periodo de estudio.

\section{Resultados de los recién nacidos}

La edad gestacional por valoración de Capurro fue de 32 a 40 semanas, con una media de 36 semanas. El Apgar promedio al minuto fue de 7 (mínimo 3, máximo 9), y a los 5 minutos fue de 9 en promedio (mínimo 5, máximo 9). El peso neonatal varió de 1600 a $3850 \mathrm{~g}$, con un promedio de $2655 \mathrm{~g}$. De las 200 pacientes, el 91\% (182) recibió un esquema de inductores de madurez pulmonar, con una media de inicio de aplicación a la semana 28 , un mínimo de inicio a las 24 semanas y un máximo a las 32 semanas.

Se identificó que 25 recién nacidos ameritaron ingreso a la unidad de cuidados intensivos neonatales; el $76 \%$ (19) tenían menos de 36 semanas y el $24 \%$ (6) tenían más de 36 semanas, por fecha de última menstruación. El 84\% de estos recién nacidos permanecieron en la unidad de cuidados intensivos entre 1 y 5 días. En la tabla 5 se muestran las complicaciones que presentaron 62 recién nacidos. Englobado en otras complicaciones, se encontraron tres casos con restricción en el crecimiento intrauterino.

El promedio de días de estancia hospitalaria en los recién nacidos fue de 6.15 días (mínimo 2, máximo 46 días); en los menores de 36 semanas de gestación, el promedio de días de estancia hospitalaria fue de 11.85, y en los de 36 o más semanas fue de 4 días.

Cabe mencionar que solo ocurrió una muerte neonatal, la cual fue secundaria a hipertensión pulmonar primaria; este embarazó se resolvió a la semana 35
Tabla 5. Complicaciones en los recién nacidos según las semanas de gestación

\begin{tabular}{lccc}
\hline Complicaciones & \multicolumn{2}{c}{$\begin{array}{c}\text { Semanas de gestación } \\
\text { según fecha de última } \\
\text { menstruación }\end{array}$} & Total \\
\cline { 2 - 3 } & Menos de 36 & Más de 36 & \\
\hline Síndrome de distrés respiratorio & 18 & 19 & 37 \\
$\begin{array}{l}\text { Taquipnea transitoria del } \\
\text { recién nacido }\end{array}$ & 4 & 6 & 10 \\
Hemorragia interventricular & 5 & 1 & 6 \\
Otras & 5 & 1 & 6 \\
Enterocolitis necrotizante & 3 & 0 & 3 \\
Total & 34 & 27 & 62 \\
\hline
\end{tabular}

mediante cirugía de urgencia por PP central total y trabajo de parto pretérmino.

\section{Discusión}

El manejo de la PP, y más cuando hay diagnóstico de acretismo, debe ser multidisciplinario y en centros de tercer nivel de salud ${ }^{16}$.

Este estudio, que incluyó 200 pacientes, mostró resultados muy similares a lo publicado por Oscanoa ${ }^{17} \mathrm{en}$ cuanto a la distribución de los diferentes tipos de inserción placentaria; este autor, al igual que nosotros, encontró la PP central total como hallazgo más común.

Pritchard, et al. ${ }^{18}$ señalan que la operación cesárea es el método aceptado para el parto en prácticamente todos los casos de PP, incluyendo los de placenta de inserción baja. En la población estudiada por nosotros se utilizó la vía abdominal en el 100\% de los casos. En los autores consultados se encuentran variaciones entre el 52.4 y el $100 \%{ }^{19-22}$.

El promedio de edad gestacional de nuestras pacientes al interrumpir el embarazo fue de 36.4 semanas; esto es más de las 35 semanas reportadas por Rojas, et al. ${ }^{23}$ en su estudio publicado en 2006. 
Cotton, et al. ${ }^{24}$ refieren una edad mayor de 36 semanas, lo cual coincide con nuestros hallazgos.

Uno de los factores predisponentes para PP, mencionado por diversos autores, es la multiparidad ${ }^{25-29}$. En nuestra casuística, el mayor porcentaje también correspondió a multíparas, con un 56\% (112).

De las 60 pacientes en que por ultrasonido se sospechó acretismo placentario, en 54 (90\%) el diagnóstico fue confirmado por patología y seis no ameritaron histerectomía obstétrica, por lo que no se confirmó el diagnóstico. Comparando nuestros resultados con los de Sánchez-García, et al. ${ }^{30}$, obtuvimos un mayor porcentaje de correlación ultrasonido-patología, pues ellos reportaron un $60.5 \%$.

El grado de invasión placentaria más común que encontramos en las 58 placentas enviadas a patología fue la placenta acreta, la cual se presentó en un porcentaje menor (27.3\%) que lo reportado por Corona y Sánchez $z^{31}$, y por Oscanoa ${ }^{17}$. La placenta increta en nuestro estudio tuvo un porcentaje del $27.3 \%$, mayor que el reportado por Boo y Merievel ${ }^{32}$, que fue del $15 \%$. Por último, en la forma percreta obtuvimos un $21 \%$, porcentaje mayor que el $10 \%$ reportado por Boo y Merievel ${ }^{32}$.

Oyelese y Smulian ${ }^{33}$ mencionan que en los EE.UU. se calcula que se producen tres muertes maternas por cada mil pacientes diagnosticadas. Rivas, et al. ${ }^{34}$ encontraron que en América Latina, a pesar de existir variedad según la región, se produce una muerte materna por cada 150 casos de PP. En nuestra casuística no se registraron muertes maternas.

De acuerdo con Flood, et al. ${ }^{35}$, las pacientes primigestas tienen un riesgo menor del $0.03 \%$ de presentar $\mathrm{PP}$, pero llama la atención que en la población estudiada encontraron un $12.5 \%$ de pacientes primigestas con PP. Vale la pena mencionar que el incremento en relación con otros estudios puede deberse a que fueron tratadas en un centro de referencia, por lo que es posible que haya un sesgo en la probabilidad real de ocurrencia en primigestas, sin dejar a un lado la idea de tratar de identificar otros factores de riesgo, y consideramos que estos casos pueden ser motivo de una investigación para encontrar los factores de riesgo o las posibles causas.

El $77 \%$ de las pacientes de nuestro estudio fueron sometidas a cirugía programada; esta cifra es mayor que la publicada por Sánchez-García, et al. ${ }^{30}$, quienes realizaron un $39.4 \%$ de cirugías programadas y un $60.5 \%$ de cirugías de urgencia. En nuestro estudio, la media de semanas de gestación en el momento de interrumpir el embarazo para la cirugía programada fue de 36.4 semanas. También observamos que la frecuencia de cirugía programada es mucho mayor en las pacientes con un embarazo de más de 36 semanas.

En nuestro estudio, en las pacientes a quienes se realizó cirugía de urgencia, las indicaciones principales fueron trabajo de parto que no respondió a inhibidores del útero, seguido de sangrado vaginal persistente, que también son las causas más comunes en la literatura ${ }^{19}$.

Comparando nuestro estudio con el publicado por Sánchez-García, et al. ${ }^{30}$, obtuvimos un mayor porcentaje de manejo conservador $62 \%$ (124) de la PP, pues ellos trataron de manera conservadora solo tres pacientes $(7.9 \%)$, y en la mayoría $(92.1 \%)$ realizaron cesárea más histerectomía obstétrica.

En cuanto al ingreso a la unidad de cuidados intensivos, se registró un menor porcentaje de ingresos (15.5\%) que el referido por Sánchez-García, et al. $^{30}(31.5 \%)$. Esto puede deberse a diferencias en los criterios de inclusión para ingresar a las pacientes de este tipo de unidad, y también a la demanda de atención para utilizar estos espacios.

La necesidad de reintervención fue del $2.5 \%$, cifra menor que en los estudios de Eller, et al. ${ }^{36}(4 \%)$ y de Sánchez-García, et al. ${ }^{30}(28 \%)$.

En las pacientes de nuestro estudio encontramos que la lesión de órgano vecino que más se presentó fue la de vejiga, en el $6.5 \%$, lo cual coincide con lo publicado por Uysal, et al. ${ }^{37}$, para quienes también fue la principal complicación.

Eller, et al ${ }^{36}$ reportaron una pérdida sanguínea promedio de $2000 \mathrm{ml}$ en los centros de atención multidisciplinaria y de $2500 \mathrm{ml}$ en los de atención convencional. En nuestra revisión, la media de sangrado fue de $1505 \mathrm{ml}$. Precisaron transfusión el 32\% de las pacientes, un porcentaje similar al publicado por Sánchez-García, et al. ${ }^{30}$.

Se realizaron un $62 \%$ (124) de procedimientos quirúrgicos conservadores, de entre los que destaca la operación cesárea $(49.5 \%)$, seguida de la cesárea más B-Lynch $(5 \%)$ y por último la cesárea más ligadura bilateral de arterias uterinas (4.5\%). Los procedimientos quirúrgicos fueron efectivos para controlar la hemorragia en el $100 \%$ de los casos, al igual que lo reportado por Fahmy ${ }^{38}$.

Comparando nuestro estudio con el publicado por Serrano ${ }^{39}$,realizamos más la ligadura de hipogástricas, la cual es comúnmente utilizada en nuestro hospital. Hay suficiente experiencia quirúrgica en la realización de este procedimiento por el 
personal médico, y es un tratamiento de elección en caso de hemorragia obstétrica grave. En esta serie no hubo pacientes en las que se dejara la placenta in situ para su remoción posterior, manejo que también ha sido documentado cuando se busca preservar la fertilidad ${ }^{40}$.Dentro del arsenal terapéutico de las pacientes con PP, y a fin de disminuir la pérdida hemática, deben considerarse las opciones de embolización de las arterias uterinas o de las hipogástricas, así como la oclusión profiláctica de la aorta abdominal con balón, procedimiento que ha mostrado disminuir significativamente el volumen de sangrado y la necesidad de histerectomía ${ }^{41}$; sin embargo, se requiere la participación de radiólogos intervencionistas, y en nuestro hospital no disponemos de los recursos para realizar estos procedimientos.

En cuanto a los resultados de los recién nacidos, no hubo diferencia significativa y todos los embarazos alcanzaron la viabilidad fetal. Las puntuaciones de Apgar en los minutos 1 y 5 fueron similares a las reportadas por McShane, et al. ${ }^{42}$, pero en nuestro estudio se presentó síndrome de distrés respiratorio en el $18.5 \%$ de los neonatos, porcentaje menor que el $21 \%$ reportado por estos autores. El porcentaje de neonatos con taquipnea transitoria del recién nacido (5\%) fue similar al publicado por McShane, et al. ${ }^{42}$.

El impacto de la PP en pacientes con restricción en el crecimiento intrauterino fue del $1.5 \%$ (3). Comparando nuestro estudio con el publicado por Neri, et al. ${ }^{43}$, obtuvimos un porcentaje mucho menor, pues ellos reportan un $10 \%$ en su estudio realizado durante 10 años en el que incluyeron 129 pacientes con diagnóstico de PP. También obtuvimos un menor porcentaje al compararlo con lo publicado por McShane, et al. ${ }^{42}$.

Respecto al riesgo de ingreso en la unidad de cuidados intensivos, Kent ${ }^{44}$ refiere una proporción del $90 \%$ para partos de emergencia y del $50 \%$ para partos programados. El ingreso en la unidad de cuidados intensivos en nuestro estudio fue del $12.5 \%$ (25), y de estos, el $36 \%$ fue para partos de emergencia y el $64 \%$ para partos programados.

Ocurrió una muerte neonatal, secundaria a hipertensión pulmonar primaria, tras un embarazó que se resolvió a la semana 35 mediante cirugía de urgencia por placenta central total y trabajo de parto pretérmino. Wen, et al. ${ }^{45}$ reportaron un $4 \%$ de muertes neonatales en 50 nacidos vivos.

Cabe señalar que en esta investigación, con la finalidad de no tener un gran sesgo en los resultados perinatales en las gestaciones de menos de 36 semanas, no se incluyeron las resueltas antes de la semana 34.

\section{Conclusiones}

Actualmente, la mortalidad materna por PP ha descendido gracias a diversos factores, como son un manejo más conservador, el uso de cesárea como método de elección para la terminación del embarazo, un control prenatal temprano y un diagnóstico oportuno, y el manejo en centros de atención especializada, con personal capacitado y los recursos humanos y materiales necesarios y suficientes.

Durante el periodo de estudio no se registraron muertes maternas relacionadas con el acretismo placentario, y un gran porcentaje de los embarazos alcanzaron las 36 semanas o más de gestación, por lo que evidenciamos que el manejo expectante de la PP, incluso con datos de acretismo, es posible y pueden alcanzarse 36 o más semanas, mejorando con ello el pronóstico fetal sin comprometer en mayor medida el pronóstico materno.

Concluimos que el manejo expectante, el diagnóstico ultrasonográfico y el tratamiento quirúrgico realizados en nuestra unidad hospitalaria ha dado resultados satisfactorios, lo que se refleja en un número mínimo de reintervenciones y complicaciones, una baja morbimortalidad neonatal y una nula mortalidad materna.

\section{Conflicto de intereses}

Los autores declaran que no existen conflictos de intereses.

\section{Financiamiento}

Los autores declaran que no hubo presupuesto de ningún tipo para la realización de esta investigación.

\section{Responsabilidades éticas}

Protección de personas y animales. Los autores declaran que para esta investigación no se han realizado experimentos en seres humanos ni en animales.

Confidencialidad de los datos. Los autores declaran que han seguido los protocolos de su centro de trabajo sobre la publicación de datos de pacientes.

Derecho a la privacidad y consentimiento informado. Los autores declaran que en este artículo no aparecen datos de pacientes. 


\section{Bibliografía}

1. Verspyck E, Douysset X, Roman H, Marret S, Marpeau L. Transecting versus avoiding incision of the anterior placenta previa during cesarean delivery. Int J Gynecol Obstet. 2014;128:44-7.

2. Silver RM. Abnormal placentation: placenta previa, vasa previa, and placenta accreta. Obstet Gynecol. 2015;126:654-68.

3. Fan D, Wu S, Wang W, Xin L, Tian G, Liu L, et al. Prevalence of placenta previa among deliveries in Mainland China: a PRISMA-compliant systematic review and meta-analysis. Medicine. 2016;95:e5107.

4. Carroli G, Cuesta C, Abalos E, Gulmezoglu AM. Epidemiology of postpartum haemorrhage: a systematic review. Best Pract Res Clin Obste Gynaecol. 2008;22:999-1012.

5. Hasegawa J, Sekizawa A, Tanaka H, Katsuragi S, Osato K, Murakoshi T, et al. Current status of pregnancy-related maternal mortality in Japan: a report from the Maternal Death Exploratory Committee in Japan. BMJ Open. 2016;6:e010304

6. Maswime S, Buchmann E. Causes and avoidable factors in maternal death due to cesarean-related hemorrhage in South Africa. Int J Gynaecol Obstet. 2016;134:320-3.

7. Say L, Chou D, Gemmill A, Tuncalp O, Moller AB, Daniels J, et al. Global causes of maternal death: a WHO systematic analysis. Lance Glob Health. 2014;2:e323-33.

8. Secretaría de Salud. Programa de Acción Específico. Salud Materna y Perinatal. 2013-2018. (Consultado en agosto de 2019) Disponible en http://cnegsr.salud.gob.mx/contenidos/descargas/SMP/SaludMaternayPerinatal_2013_2018.pdf

9. Haghenbeck-Altamirano FJ, Leis-Márquez T, Ayala-Yáñez R, Juárez-García LC, García-Moreno C. Diagnóstico antenatal de acretismo-percretismo placentario. Ginecol Obstet Mex. 2013;81:259-71.

10. American College of Obstetricians and Gynecologists. Obstetric care consensus. Placenta accreta spectrum. Obstet Gynecol. 2018;132:259-75.

11. Jolley JA, Nageotte MP, Wing DA, Shrivastava VK. Management of placenta accreta: a survey of maternal-fetal medicine practitioners. J Matern Fetal Neonatal Med. 2012;25:756-60.

12. Esakoff TF, Sparks TN, Kaimal AJ, Kim LH, Feldstein VA, Goldstein RB, et al. Diagnosis and morbidity of placenta accreta. Ultrasound Obstet Gynecol. 2011;37:324-7

13. Esakoff TF, Handler SJ, Granados JM, Caughey AB. PAMUS: placenta accreta management across the United States. J Matern Fetal Neonatal Med. 2012;25:761-5.

14. Sumano-Ziga E, Veloz-Martínez MG, Vázquez-Rodríguez JG, Becerra-Alcantara GI, Jimenez-Vieyra CR. Histerectomía programada vs. histerectomía de urgencia en pacientes con placenta acreta, en una unidad médica de alta especialidad. Cir Cir. 2015;83:303-8.

15. Vergani P, Ornaghi S, Pozzi I, Beretta P, Russi FM, Follesa I, et al. Placenta previa: distance to internal os and mode of delivery. Am J Obstet Gynecol. 2009;201:266.e1-5.

16. Consejo de Salubridad General. Guía de práctica clínica para el diagnóstico y manejo de anomalías en la inserción placentaria y vasos sanguíneos fetales. México: Secretaría de Salud; 2013. (Consultado en agosto de 2019) Disponible en: www.cenetec-difusion.com/CMGPC/ GPC-IMSS-589-19/ER.pdf

17. Oscanoa A. Placenta previa. Instituto Especializado Materno Fetal. Rev Per Gineco Obstetr. 2005:51:2-9.

18. Pritchard J, McDonald P, Gant N. Williams Obstetricia. 3. ${ }^{a}$ ed. Barcelona: Salvat; 1998. p. 693-727.

19. Fachin De Boni J, Peña G. Hemorragia intra y posparto. Rev Obstet Ginecol Venez. 1963;23:187-201.

20. Lira J, Cabral S, Argueta M, Karchmer S, Ibasguengoitia F. Placenta previa. Repercusiones maternas y perinatales. Análisis de 170 casos. Ginecol Obstet Mex. 1995;63:175-80.

21. Chesvenak F, Lee Y, Hendler M, Monoson R, Berkowitz R. Role of attempted vaginal delivery in the management of placenta previa. Am $J$ Obstet Gynecol. 1984;64:798-801.
22. Jauniaux E, Alfirevic Z, Bhide A, Belfort MA, Burton GJ, Collins SL, et al. Placenta praevia and placenta accreta: diagnosis and management: green-top guideline No. 27a. BJOG. 2019;126:e1-e48.

23. Rojas F, Kuzmar L, Ferreira F, Abdalla M. Diagnóstico y manejo quirúrgico de placenta acreta: presentación de un caso y revisión de la literatura. Rev Colomb Obstet Ginecol. 2006;57:123-8.

24. Cotton D, Read J, Paul R, Quilligan E. The conservative aggressive management of placenta previa. Am J Obstet Gynecol. 1980;137:687-95.

25. Shamshirsaz A, Fox K, Salmanian B, Díaz-Arrastia C, Lee W, Baker B, et al. Maternal morbidity in patients with morbidly adherent placenta treated with and without a standardized multidisciplinary approach. Am J Obstet Gynecol. 2015;212:218.e1-9.

26. Ananth C, Smulian J, Vintzileos A. The association of placenta previa with history of cesarean delivery and abortion: metaanalysis. Am J Obstet Gynecol. 1977;177:1071-8.

27. Niswander K, Friedman E, Hooker D, Pietrowski H, Westphal M. Fetal morbidity following potentially anoxigenic obstetric conditions. II. Placenta previa. Am J Obstet Gynecol. 1966;95:846-52.

28. Taylor V, Kramer M, Vaughan TH, Peacock S. Placenta previa and prior cesarean delivery: how strong is the association? Am J Obstet Gynecol. 1994;84:55-7.

29. Fan D, Xia Q, Liu L, Wu S, Tian G, Wang W, et al. The incidence of postpartum hemorrhage in pregnant women with placenta previa: a systematic review and meta-analysis. PLoS One. 2017;12:e0170194.

30. Sánchez-García BF, Corona-Gutiérrez AA, Sánchez-Peña R, Panduro-Barón JG, Barrios-Prieto E, González-Moreno J. Acretismo placentario en el Hospital Civil de Guadalajara Dr. Juan I. Menchaca. Rev Med MD. 2013;4:240-4.

31. Corona GAA, Sánchez PR. Guía práctica clínica placenta acreta. Hospital Civil Juan I. Menchaca. Guadalajara; 2010.

32. Boo G, Merievel P. Placenta accreta. Encycl Med Chir (Editions Scientifiques et Medicales Elsevier SAS, Paris-France), Obstetrique, E-5069-A-30, 2003:1-12.

33. Oyelese Y, Smulian JC. Placenta previa, placenta accreta, and vasa previa. Obstet Gynecol. 2006;107:927-41.

34. Rivas M, López JR, Capretta D, Colmenares B, Silva D, Alvarado S. Placenta previa. Estudio retrospectivo 1988-1992. Rev Obstet Ginecol Venez. 2001;61:217-22.

35. Flood KM, Said S, Geary M, Robson M, Fitzpatrick C, Malone FD. Changing trends in peripartum hysterectomy over the last 4 decades. Am J Obstet Gynecol. 2009;200:632.e1-6.

36. Eller AG, Bennett MA, Shashiner M, Masheter C, Soisson AP, Dodson M, et al. Maternal morbidity in cases of placenta accreta managed by a multidisciplinary care team compared with standard obstetric care. Am J Obstet Gynecol. 2011;117:331-7.

37. Uysal D, Cokmez H, Aydin C, Ciftpinar T. Emergency peripartum hysterectomy: a retrospective study in a tertiary care hospital in Turkey from 2007 to 2015. J Pak Med Assoc. 2018;68:3.

38. Fahmy K. Uterine artery ligation to control postpartum hemorrhage. Int J Gynecol Obstet. 1987;25:363-7.

39. Serrano M. Comparación de dos técnicas quirúrgicas conservadoras para el tratamiento de la hemorragia obstétrica. Rev Esp Med Quir. 2013;18:100-7.

40. MacGibbon A, lus YM. Conservative management of abnormally invasive placenta previa after midtrimester foetal demise. Case Rep Obstet Gynecol. 2018;2018:7478437.

41. Chen L, Wang X, Wang H, Li Q, Shan N, Qi H. Clinical evaluation of prophylactic abdominal aortic balloon occlusion in patients with placenta accreta: a systematic review and meta-analysis. BMC Pregnancy Childbirth. 2019;19:30.

42. McShane P, Heyl P, Epstein MD. Maternal and perinatal morbidity resulting from placenta previa. Am J Obstet Gynecol. 1985:65:176-80.

43. Neri A, Gorodesky I, Bahary C, Ovadia Y. Impact of placenta praevia on intrauterine fetal growth. Isr J Med Sci. 1980;16:429-32.

44. Kent A. Management of placenta accreta. Rev Obstet Gynecol. 2009;2:127-8.

45. Wen S, Demissie K, Liu S, Marcoux S, Kramer M. Placenta previa and male sex at birth: results from a population-based study. Paediatr Perinat Epidemiol. 2000;14:300-4. 